\title{
AN ARITHMETICAL PROPERTY OF RECURRING SERIES OF THE SECOND ORDER*
}

\author{
BY MORGAN WARD
}

1. Statement of Property. Let us denote by

$$
\left(W_{n}\right): W_{0}, W_{1}, W_{2}, \cdots, W_{n}, \cdots,
$$

a sequence of rational integers satisfying the difference equation

$$
\Omega_{n+2}=P \Omega_{n+1}-Q \Omega_{n}, \quad(P, Q \text { rational integers }),
$$

and let $p$ be an odd prime dividing neither $Q$ nor $P^{2}-4 Q$ $=(\alpha-\beta)^{2}$, the discriminant of the polynomial

$$
x^{2}-P x+Q=(x-\alpha)(x-\beta)
$$

associated with (1). $\dagger$

We write as usual $U_{n}=\left(\alpha^{n}-\beta^{n}\right) /(\alpha-\beta), V_{n}=\alpha^{n}+\beta^{n}$ for the two Lucas functions built upon the roots $\alpha$ and $\beta$ of (2).

The distribution of the multiples of $p$ in the corresponding sequences $(U)_{n}$ and $(V)_{n}$ is well known: namely, multiples of $p$ always occur in $(U)_{n}$; more specifically, $U_{n} \equiv 0(\bmod p)$ when and only when $n \equiv 0(\bmod \tau)$, where $\tau$ is the restricted period $\ddagger$ of $(U)_{n}$ modulo $p$. In the sequence $(V)_{n}$, multiples of $p$ occur when and only when $\tau$ is even. In this case, $V_{n} \equiv 0(\bmod p)$ when and only when $n \equiv 0(\bmod \tau / 2), n \neq 0(\bmod \tau)$.

For the sequences $(U)_{n}$ and $(V)_{n}$ then, we know not only when multiples of $p$ will occur, but where multiples of $p$ will occur. Under the assumption that $\tau$ is odd, I propose to obtain a criterion which reduces the problem of determining when multiples of $p$ will appear in any sequence $(W)_{n}$ (specified only by its two initial values $W_{0}$ and $W_{1}$ ) to the more fundamental (unsolved) problem of determining the characteristic number $\ddagger$ and restricted period $\ddagger$ of the Lucas functions associated with any given quadratic polynomial of the form (2).

* Presented to the Society, June 20, 1934.

$\dagger$ The excluded values of $p$ are evidently trivial for the theorem that follows.

¥ For definitions of these terms, see my Note on the period of a mark in a finite field, this Bulletin, vol. 40 (1934), pp. 279-281. 
In fact, let $P^{\prime}$ and $Q^{\prime}$ be rational integers satisfying the congruences

$$
\begin{aligned}
P^{\prime} & \equiv W_{0}(\bmod p), \\
\left(4 Q-P^{2}\right) Q^{\prime} & \equiv W_{1}^{2}-W_{0} W_{1} P+W_{0}^{2} Q(\bmod p),
\end{aligned}
$$

and let $U_{n}^{\prime}=\left(\alpha^{\prime n}-\beta^{\prime n}\right) /\left(\alpha^{\prime}-\beta^{\prime}\right)$ be the Lucas function associated with the polynomial $x^{2}-P^{\prime} x+Q^{\prime}=\left(x-\alpha^{\prime}\right)\left(x-\beta^{\prime}\right)$. Then $a$ necessary and sufficient condition that the sequence $(W)_{n}$ should contain multiples of $p$ is that the restricted period of $U_{n}^{\prime}$ modulo $p$ should be an even divisor of $2 \tau$.

2. Illustration. As a numerical illustration, take $P=1, Q=-1$, $p=89, W_{0}=1, W_{1}=4$. The sequence $(U)_{n}$ is then the familiar Fibonacci series

$$
0,1,1,2,3,5,8,13,21,34,55,89, \cdots
$$

so that $\tau=11$. The congruences (3) become $P^{\prime} \equiv 1(\bmod 89)$, $-5 Q^{\prime} \equiv 11(\bmod 89)$, so that we may take $P^{\prime}=1, Q^{\prime}=-20$. The Lucas sequence $\left(U^{\prime}\right)_{n}$ for $\Omega_{n+2}=\Omega_{n+1}+20 \Omega_{n}$ runs $0,1,1,21,41$, $461,1281,10501, \cdots$ and by actual computation we find that $U_{22}^{\prime} \equiv 69(\bmod 89)$. Hence the restricted period of $\left(U^{\prime}\right)_{n}$ modulo 89 is not an even divisor of $2 \tau=22$, and we conclude that all elements of the sequence $1,4,5,9,14,23,37, \cdots$ are prime to 89 , as may be easily verified.

3. A Preliminary Identity. My proof of this result is based upon a well known identity in the theory of partitions discovered by Euler, ${ }^{*}$ which we formulate as follows.

Let $q$ be any complex number,

$$
[n]\left\{\begin{array}{l}
=\left(q^{n}-1\right) /(q-1), q \neq 1, \\
=\lim _{q \rightarrow 1}\left(q^{n}-1\right) /(q-1)=n, q=1 .
\end{array}\right.
$$

Writing $[n]$ ! for $[1][2] \cdots[n],[0] !=1$, we see that the basic bi-

* Introductio in Analysin Infinitorum, 1748, Chapter VII; Netto, Combinatorik, 2d ed., 1927, p. 143. 
nomial coefficient ${ }^{*}(n ; r)$ is defined by $(n ; r)=[n] ! /\{[n-r] ![r] !\}$. This expression, as Gauss showed, $\dagger$ is a polynomial in $q$ which reduces to the ordinary binomial coefficient when $q=1$. The identity in question may now be written as follows:

(4) $\sum_{r=0}^{\tau}(\tau ; r) q^{r(r+1) / 2} z^{r}=(1+q z)\left(1+q^{2} z\right) \cdots\left(1+q^{\tau} z\right)$.

4. Proof. The general term of the sequence $(W)_{n}$ may be expressed in the form

$$
W_{n}=W_{0} U_{n+1}+\left(W_{1}-P W_{0}\right) U_{n} .
$$

Thus the restricted period of $(W)_{n}$ modulo $p$ is a divisor of the restricted period $\tau$ modulo $p$ of the Lucas function $U_{n}$. Therefore the sequence $(W)_{n}$ will contain terms divisible by $p$ when and only when the rational integer

$$
\mathfrak{W}=\prod_{n=1}^{r} W_{n}
$$

is divisible by $p$.

Now $W_{n}$ can also be expressed in the form $W_{n}=A \alpha^{n}+B \beta^{n}$, where the constants $A$ and $B$ are determined by

$$
W_{0}=A+B, \quad W_{1}=A \alpha+B \beta .
$$

If we let $\ddagger \beta / \alpha=q, B / A=z$, we may write $W_{n}=A \alpha^{n}\left(1+q^{n} z\right)$. Therefore by (5) and (4),

$$
\begin{aligned}
\mathfrak{W} & =\prod_{n=1}^{\tau} A \alpha^{n}\left(1+q^{n} z\right)=A^{\tau} \alpha^{\tau(\tau+1) / 2} \prod_{n=1}^{\tau}\left(1+q^{n} z\right) \\
& =A^{\tau} \alpha^{\tau(\tau+1) / 2} \sum_{r=0}^{\tau}(\tau ; r) q^{r(r+1) / 2} z^{r} .
\end{aligned}
$$

* This terminology is due to F. H. Jackson who in recent years has made an extensive study of the basic numbers $[n]$. (See, for example, Proceedings of the London Mathematical Society, (2), vol. 1 (1903-04), pp. 63-68; Proceedings of the Royal Society, (A), vol. 76 (1905), pp. 127-144.)

$\dagger$ Summatio quarundam Serierum Singularium, 1808; Works, vol. 2, p.16.

$\ddagger$ If $A$ and $B$ are rational integers modulo $p$, they cannot both be congruent to zero, and we take for $A$ that one which is incongruent to zero modulo $p$. We have $\alpha \neq 0 \bmod p$, since $p$ was assumed prime to $Q$. 
Now $[n]=\left(q^{n}-1\right) /(q-1)=\left(\alpha^{n}-\beta^{n}\right) /\left[(\alpha-\beta) \alpha^{n-1}\right]=\alpha^{-n+1} U_{n}$. Hence

$$
(n ; r)=U_{n} U_{n-1} \cdots U_{n-r+1} /\left(U_{1} U_{2} \cdots U_{r} \alpha^{-(n-r) r}\right) .
$$

But the first $\tau-1$ of the numbers $U_{1}, U_{2}, \cdots, U_{\tau}$ are prime to $p$, while $U_{\tau}$ is divisible by $p$. Hence $(\tau ; r) \equiv 0(\bmod p)$ unless $r=0$ or $r=\tau$, when it equals one. We therefore obtain from (7) the congruence

$\mathfrak{W} \equiv A^{\tau} \alpha^{\tau(\tau+1) / 2}\left(1+q^{\tau(\tau+1) / 2} z^{\tau}\right) \equiv A^{\tau} \alpha^{\tau(\tau+1) / 2}+B^{\tau} \beta^{\tau(\tau+1) / 2}(\bmod p)$.

Now $\alpha^{n}=U_{n} \alpha-Q U_{n-1}, \beta^{n}=U_{n} \beta-Q U_{n-1}$. Therefore since $\tau$ is odd, ${ }^{*}$

$$
\begin{aligned}
& \alpha^{\tau(\tau+1) / 2} \equiv\left(U_{\tau} \alpha-Q U_{\tau-1}\right)^{(\tau+1) / 2} \equiv\left(-Q U_{\tau-1}\right)^{(\tau+1) / 2}(\bmod p), \\
& \beta^{\tau(\tau+1) / 2} \equiv\left(-Q U_{\tau-1}\right)^{(r+1) / 2}(\bmod p),
\end{aligned}
$$

and

$$
\mathfrak{W} \equiv\left(-Q U_{\tau-1}\right)^{(\tau+1) / 2}\left(A^{\tau}+B^{\tau}\right)(\bmod p) .
$$

Hence $\mathfrak{W} \equiv 0(\bmod p)$ when and only when $A^{r}+B^{r} \equiv 0(\bmod p)$.

Finally, write $\alpha^{\prime}, \beta^{\prime}$ for $A, B$. Then $A^{n}+B^{n}=V_{n}^{\prime}$, the Lucas function associated with the quadratic polynomial $x^{2}-P^{\prime} x+Q^{\prime}$ $=\left(x-\alpha^{\prime}\right)\left(x-\beta^{\prime}\right)$.

On referring back to (6) and recalling that $p$ is prime to $P^{2}-4 Q$, we find that we may assign to $P^{\prime}$ and $Q^{\prime}$ the rational integral values specified by the congruences (3). Our theorem now follows immediately from the laws of apparition for multiples of $p$ in the Lucas functions stated in section 1 as applied to the sequence $\left(V^{\prime}\right)_{n}$.

\section{California Institute of Technology}

* It is at precisely this point that the assumption that $\tau$ is odd becomes vital. For if we assume that $\tau$ is even, we obtain in place of (8) the barren result $\mathfrak{W}=\left(U_{\tau+1}\right)^{\tau / 2}\left(A^{\tau} \alpha^{\tau / 2}+B^{\tau} \beta^{\tau / 2}\right)(\bmod p)$. 